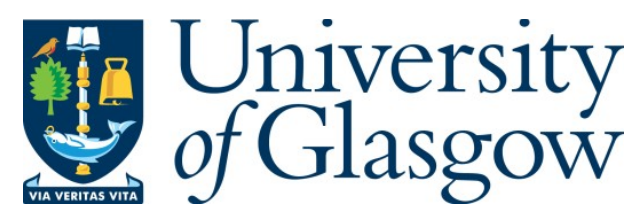

Boychev, K., Barakos, G.N., Steijl, R. and Shaw, S. (2021) Simulations of flows around complex and simplified supersonic store geometries at high incidence angles using statistical and scale-resolving turbulence models. In: AIAA SCITECH 2022 Forum, San Diego, CA \& Virtual, 3-7 January 2022, ISBN 9781624106316

(doi: $\underline{10.2514 / 6.2022-1686)}$

This is the Author Accepted Manuscript.

There may be differences between this version and the published version. You are advised to consult the publisher's version if you wish to cite from it.

http://eprints.gla.ac.uk/260571/

Deposited on: 13 December 2021

Enlighten - Research publications by members of the University of Glasgow http://eprints.gla.ac.uk 


\title{
Simulations of supersonic store geometries at high incidence angles using statistical and scale-resolving models
}

\author{
K. Boychev*, G.N. Barakos ${ }^{\dagger}$, R. Steij ${ }^{\ddagger}$ \\ CFD Laboratory, School of Engineering, University of Glasgow, G128QQ, Glasgow UK \\ S. Shaw ${ }^{\S}$ \\ MBDA UK Ltd, Filton, Bristol, BS347QW, UK
}

\begin{abstract}
Predicting the flowfield around a supersonic store at a high incidence angle is challenging due to the presence of vortices and shocks that interact with each other. The complexity of the problem is further increased by the presence of wing-body and wing-tail junctions giving rise to secondary flows. Given that the flow is turbulent, linear eddy-viscosity turbulence models are unable to account for the secondary flows and are often more dissipative than their non-linear counterparts. The high incidence angle further increases the complexity. This work investigates the effect of grid refinement and turbulence modelling on three store configurations - one with wings and fins, one without fins and wings, and one with wings only. The in-house CFD solver of the University of Glasgow is used to perform simulations at different angles of incidence and roll. Grids consisting of approximately $80 \times 10^{6}$ cells or less were found to be inadequate to capture the flow features. This shows that even if a high-order spatial method is employed, a grid of sufficient density must be used to accurately capture the aerodynamic loads of the store. In addition, grid converged results were difficult to obtain for the full configuration due to the interaction of the wing vortices with the store's fins. Improved convergence was observed for the simplified store configurations. This further showed that the difficulty in grid convergence is related to the wing vortex interactions with the store's fins.
\end{abstract}

$\begin{array}{lll} & & \\ \text { Latin } & = & \text { Width of numerical domain } \\ W & = & \text { Height of numerical domain } \\ H & = & \text { Turbulent kinetic energy } \\ k & = & \text { Length of store } \\ L & = & \text { Mach number } \\ M & = & \text { Static pressure } \\ p & = & \text { Flux residual vector } \\ \mathbf{R} & = & \text { Reynolds number } \\ R e & = & \text { Time } \\ t & = & \text { Velocity vector } \\ \mathbf{V}=(u, v, w)^{T} & =\text { Velocity vector magnitude } \\ V=|\mathbf{V}| & =\text { Volume of cell i,j, } \mathrm{k} \\ \forall_{i, j, k} & =\text { Vector of conservative flow variables } \\ \mathbf{W} & =\text { Position vector } \\ \mathbf{x}=(x, y, z)^{T} & =\text { Diameter } \\ d & =\text { Area } \\ S & =\text { Aerodynamic coefficient in the } x \text {-direction } \\ C_{X} & =\text { Aerodynamic coefficient in the } y \text {-direction } \\ C_{Y} & \end{array}$

\footnotetext{
*PhD Student, CFD Laboratory, School of Engineering, Email: k.boychev.1@ research.gla.ac.uk

${ }^{\dagger}$ Professor, MAIAA, MRAeS, CFD Laboratory, School of Engineering, Email: George.Barakos@glasgow.ac.uk

†Senior Lecturer, CFD Laboratory, School of Engineering, Email: Rene.Steijl@glasgow.ac.uk

${ }^{\S}$ Senior Principal Aerodynamicist, MBDA UK Ltd, Email: scott.shaw@ @bda-systems.com
} 


$\begin{array}{ll}C_{Z} & =\text { Aerodynamic coefficient in the } z \text {-direction } \\ C_{l} & =\text { Aerodynamic coefficient about the } x \text {-axis (rolling moment) } \\ C_{m} & =\text { Aerodynamic coefficient about the } y \text {-axis (pitching moment) } \\ C_{n} & =\text { Aerodynamic coefficient about the } z \text {-axis (yawing moment) }\end{array}$

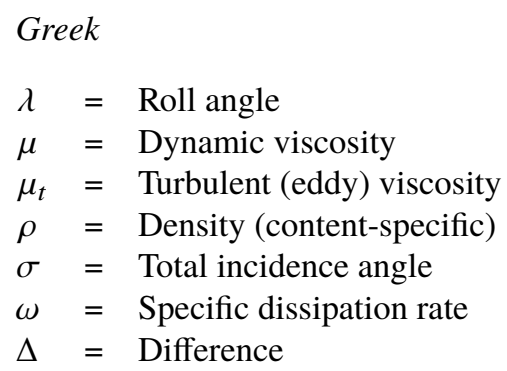

Subscripts and superscripts

$(.)_{r e f}=$ Reference quantity

Acronyms

AVT $=$ Applied Vehicle Technology

NATO $=$ North Atlantic Treaty Organisation

EARSM $=$ Explicit Algebraic Reynolds Stress Model

QCR = Quadratic Constitutive Relation

RANS $=$ Reynolds Averaged Navier Stokes

SST $=$ Shear Stress Transport

GCI $=$ Grid Convergence Index

TKE $=$ Turbulent Kinetic Energy

RET = eddy to molecular viscosity ratio

\section{Introduction}

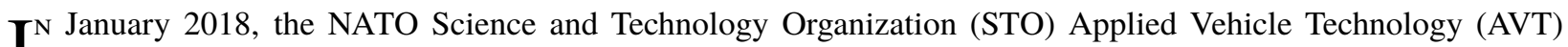
I panel established a Task Group identified as AVT-316 (Vortex Interaction Effects Relevant to Military Air Vehicle Performance). The Task Group was split into two facets: an Aircraft Facet and a Missile Facet, each focusing on the vortex interactions associated with airframes of direct interest to NATO. The Missile Facet was established to (i) Assess the current capabilities of CFD to predict missile aerodynamic characteristics for flows containing multiple vortex interactions; (ii) Share and seek to learn from comparable experience of applying CFD to other classes of NATO vehicles (combat aircraft, in particular); and (iii) Consolidate lessons learned and any attendant future requirements [1]. This paper is one of a series being presented at this conference to provide a technical overview of the activities and accomplishments of the AVT-316 Missile Facet [2-11]. The work is still ongoing: a final output, constituting a more detailed and consolidated technical record, will be published by NATO STO towards the end of 2022.

With the continued increase of computing power over the last decade, computational fluid dynamic (CFD) methods are becoming an indispensable tool during the design and development stages of air vehicles. CFD methods are especially useful in situations where the flows under investigation are difficult to measure experimentally. Many advances have been made in CFD over the past 25 years including the development of high-resolution numerical schemes and improved turbulence models capable of capturing complex flow physics. Nevertheless, there are still flows that present challenges to the state of the art CFD methods. Examples are flows around bodies of revolution and flows around delta wings at high incidence angle. Such flows are vortex dominated and, if supersonic, they contain shocks and vortex shock interactions. Historically, the difficulty in accurately predicting such flows with CFD methods stems from the fine grids 
required to capture the vortex structures. In addition complex geometries featuring sharp corners further, increase the complexity of the problem. Standard linear turbulence models such as the k- $\omega$ BSL and k- $\omega$ SST are inherently unable to resolve for the secondary flows arising near leading edges. The generation of secondary corner flows is governed by the normal Reynolds stress anisotropy in the vicinity of the corner. As linear models are isotropic, they fail to generate secondary flows near corners. In the context of the current AVT-316 exercise, (see figure 1), sharp corners are present at the locations where the wings or fins meet the store body.

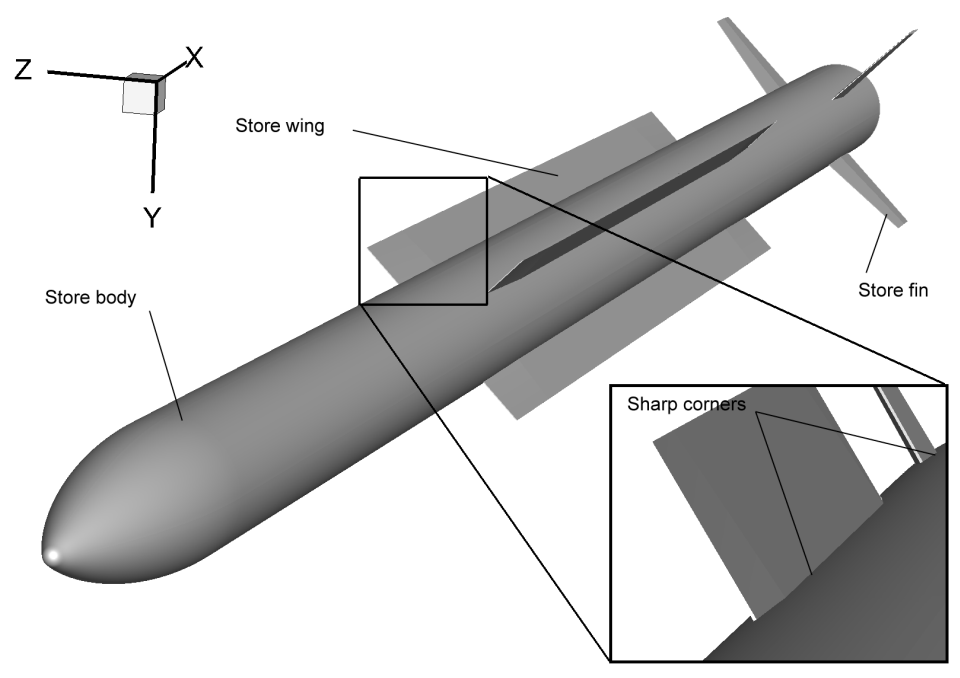

Fig. 1 Store geometry and sharp corners which give rise to the formation of secondary flows (contra-rotating vortices)

In addition, linear models predict excessive levels of eddy viscosity at the vortex cores and near shear layers. This is not favourable as it changes the underlying flow physics. Non-linear models and scale-resolving methods can be used to remedy this shortcoming by offering greater modelling fidelity, however, they come with their own problems. The convergence speed can decrease due to the non-linearity of the models and the well-known dependence of the DES [12] scale resolving method to the grid size. The present work investigates the effect of non-linear turbulence models and scale-resolving methods on the ability of CFD methods to accurately predict a complex vortically-dominated supersonic flow around a generic store geometry. The effect of the non-linear k- $\omega$ EARSM and k- $\omega$ SST SAS models are considered. The latter model was considered due to its formulation being independent of the local grid size, in comparison to DES. First simulations of the full geometry, featuring wings and fins, are presented and the effect of turbulence modelling and grid convergence are discussed. In a second step, simplified geometries of the store were simulated to investigate the lack of grid convergence observed for the full geometry.

\section{Numerical method}

The Helicopter Multi-Block (HMB3) [13, 14] code is used in the present work. HMB3 solves the Unsteady Reynolds Averaged Navier-Stokes (URANS) equations in integral form using the Arbitrary Lagrangian-Eulerian (ALE) formulation for time-dependent domains, which may include moving boundaries. The Navier-Stokes equations are discretised using a cell-centred finite volume approach on a multi-block grid. The spatial discretisation of these equations leads to a set of ordinary differential equations in time

$$
\frac{d}{d t}\left(\mathbf{W}_{i, j, k} \forall_{i, j, k}\right)=-\mathbf{R}_{i, j, k}\left(\mathbf{W}_{i, j, k}\right),
$$

where $i, j, k$ represent the cell index, $\mathbf{W}_{i, j, k}$ and $\mathbf{R}_{i, j, k}$ are the vector of conservative flow variables and flux residual respectively, and $\forall_{i, j, k}$ is the volume of the cell $i, j, k$. To evaluate the convective fluxes the Osher [15] approximate Riemann solver is used, while the viscous terms are discretised using a second-order central differencing spatial 
discretisation. The Monotone Upstream-centered Schemes for Conservation Laws, which is referred to in the literature as the MUSCL approach and developed by Leer [16], is used to provide high-order accuracy in space. The HMB3 solver uses the alternative form of the Albada limiter [17] being activated in regions where large gradients are encountered mainly due to shock waves, avoiding the non-physical spurious oscillations. An implicit dual-time stepping method is employed to perform the temporal integration, where the solution is marching in pseudo-time iterations to achieve a fast convergence, which is solved using a first-order backward difference. The linearised system of equations is solved using the Generalised Conjugate Gradient method with a Block Incomplete Lower-Upper (BILU) factorisation as a pre-conditioner [18]. To allow an easy sharing of the calculation load for a parallel job, multi-block structured grids are used. Various turbulence models are available in the HMB3 solver, including several one-equation, two-equation, three-equation, and four-equation turbulence models. Furthermore, Large-eddy Simulation (LES), Detached-eddy Simulation (DES), and Delayed-Detached-eddy Simulation (DDES) are also available. In the present work the fully-turbulent $k-\omega$ SST by Menter [19] and $k-\omega$ EARSM by Hellsten [20] turbulence models are used.

\section{A. Turbulence modelling}

In this work, the Scale Adaptive Simulation (SAS), the k- $\omega$ SST turbulence model by Menter [19], and the k- $\omega$ EARSM turbulence model by Hellsten [20] are used. A brief description of each model follows.

\section{Scale Adaptive Simulation (SAS)}

In the original formulation of the Detached eddy Simulation proposed by Spalart [12], the turbulent length scale computed from the turbulence model is compared to the local grid spacing. The decrease of the eddy viscosity, which allows the model to switch to scale resolving mode, is controlled entirely by the destruction term $\left(D_{k}=\beta \rho \omega k F_{D E S}\right)$ for the turbulent kinetic energy. Menter [] showed that a destruction term for the eddy viscosity can be formulated without the explicit grid dependency in his KE1E model. The destruction term is based on the von Karman length scale (which acts as an integral length scale in boundary layer regions) and is given by:

$$
\begin{gathered}
D=c_{2}\left(\frac{v_{t}}{l_{v k}}\right)^{2}, \\
l_{v k}=\kappa\left|\frac{\frac{\partial \bar{u}}{\partial y}}{\frac{\partial^{2} \bar{u}}{\partial y^{2}}}\right|,
\end{gathered}
$$

where $\bar{u}$ is the velocity parallel to the wall and $\mathrm{y}$ is the wall-normal coordinate. For general three-dimensional calculations including modifications to avoid the singularity for vanishing mean strain-rate Menter et al. [21] used the following invariant formulation of the von Karman length scale

$$
l_{v k}=\kappa \sqrt{\frac{\frac{\partial \bar{u}_{i}}{\partial y_{j}} \frac{\partial \bar{u}_{i}}{\partial y_{j}}}{\frac{\partial^{2} \bar{u}_{l}}{\partial y_{m}^{2}} \frac{\partial^{2} \bar{u}_{m}}{\partial y_{l}^{2}}}}
$$

and proposed the following modification to the KE1E model:

$$
\begin{array}{r}
\widetilde{l_{v k}}=\max \left(l_{v k}, C_{S A S} \widetilde{\Delta}\right), \\
\widetilde{\Delta}=\max (\Delta x, \Delta y, \Delta z), \\
C_{S A S}=0.6 .
\end{array}
$$

It was found that the above length scale introduces a dynamic behaviour in the KE1E model. The SAS formulation was extended to the k- $\omega$ SST model by Menter and Egorov [22]. In the model an additional destruction term $Q_{S A S}$ in the equation for the specific dissipation rate $-\omega$ is present.

$$
Q_{S A S}=\max \left[\zeta_{2} \kappa S^{2}\left(\frac{l_{R A N S}}{\widetilde{l_{v K}}}\right)^{2}-C \frac{2 k}{\sigma_{\Phi}} \max \left(\frac{|\nabla \omega|^{2}}{\omega^{2}}, \frac{|\nabla k|^{2}}{k^{2}}\right), 0\right],
$$


where

$$
\begin{array}{r}
\zeta_{2}=3.51, \\
\sigma_{\Phi}=2 / 3, \\
C=2, \\
\kappa=0.41, \\
{ }^{-1 / 4} \frac{\sqrt{k}}{\omega}, \\
l_{R A N S}=C_{\mu}=\max \left(\frac{\kappa S}{\left|\nabla^{2} U\right|}, 0.26(\Delta x \Delta y \Delta z)^{1 / 3}\right), \\
S=\sqrt{2 S_{i j} S_{i j}} .
\end{array}
$$

When $Q_{S A S}=0$ the resulting mode is RANS (attached flows). When $Q_{S A S}>0$ the model switches to scale resolving mode providing LES-like behaviour.

\section{Explicit Algebraic Reynolds Stress (EARSM) model}

The Explicit Algebraic Reynolds Stress k- $\omega$ model proposed by Hellsten [20] is derived from Menter's Baseline k- $\omega$ model [19] but uses the explicit algebraic Reynolds-stress model of Wallin and Johansson [13] as a constitutive model for the turbulent stress tensor (the constant coefficients are also re-calibrated). The turbulent stress tensor is given by:

$$
\tau_{i j}^{R A N S}=\tau_{i j}^{R A N S}-a_{i j} \rho k,
$$

where $\tau_{i j}^{R A N S}$ is the linear stress tensor (from the Boussinesq approximation) and $a_{i j}$ is the non-linear stress tensor (from the constitutive model). A similar approach is used by models which employ the Quadratic Constitutive Relation (QCR) proposed by Spalart [12]. Full details about the k- $\omega$ EARSM model can be found on the NASA turbulence modelling resource website [23].

\section{B. Convergence analysis}

Methods for evaluating the temporal and spatial convergence of CFD simulations (based on Richardson's extrapolation) were proposed by Roache [24, 25]. The grid convergence index or GCI [24, 25] presents a simple method for uniform reporting of grid convergence studies without any restriction to grid doubling. The GCI is based on generalised Richardson extrapolation involving the comparison of discrete solutions at two different grid spacings. The error in a fine grid solution $f_{1}$ can be obtained by comparing it to the solution on a coarse grid $f_{2}$ and is defined as:

$$
E^{\text {fine }}=\frac{\epsilon_{12}}{\left(1-r^{p}\right)},
$$

where $\epsilon_{12}=f_{2}-f_{1}, r$ is the refinement ratio and $p$ is the observed order of accuracy. A second-order numerical method will have $p=2$, however, due to factors including but not limited to the grid the observed order of accuracy will be less. To account for the uncertainty in the generalised Richardson-based error estimates and to put the grid convergence studies on the same basis as grid doubling with a second-order numerical algorithm Roache [24, 25] incorporated a safety factor and defined the GCI as:

$$
G C I^{\text {fine }}=F_{S}\left|E^{\text {fine }}\right|,
$$

where $F_{S}$ is a safety factor. The $\mathrm{GCI}$ is not an error estimator but $F_{S}$ times the error estimator, representing error bands in a loose statistical sense. A value of $F_{s}=3$ for two grids and $F_{s}=1.25$ for three grids was recommended by Roache [25]. For three grids, having solutions $f_{1}, f_{2}$, and $f_{3}$, where $f_{1}$ corresponds to the solution on the finest grid, the observed order of convergence, $p$, is given by: 


$$
\frac{\epsilon_{23}}{\left(r_{23}^{p}-1\right)}=r_{12}^{p}\left[\frac{\epsilon_{12}}{\left(r_{12}^{p}-1\right)}\right] .
$$

As the above equations are transcendental in $p$, an iterative method is required to solve it. For well-behaved solutions simple substitution iteration with a relaxation factor of $\omega=0.5$ works well.

$$
\begin{array}{r}
\beta=\frac{\left(r_{12}^{p^{i}}-1\right)}{\left(r_{23}^{p^{i}}-1\right)} \frac{\epsilon_{23}}{\epsilon_{12}} \\
p^{i+1}=\omega p^{i}+(1-\omega) \frac{\ln (\beta)}{\ln \left(r_{12}\right)} ;
\end{array}
$$

This approach is used here to determine whether or not grid convergence is achieved.

\section{Numerical setup}

In this work, three geometries are considered. The geometries were based on the geometry in the NATO AVT-316 activity [1, 26]. The first geometry represents a full store, the second a store body only and the third a store body with wings. The geometries are referred to as configuration A, B, and C. The length of the store is $L_{r e f}=3.45 \mathrm{~m}$ and the diameter $d_{\text {ref }}=0.15 \mathrm{~m}$. Figure 2 below shows the configurations. The loads are reported in a right-hand coordinate system with an origin located at $x / L_{r e f}=0.5$.

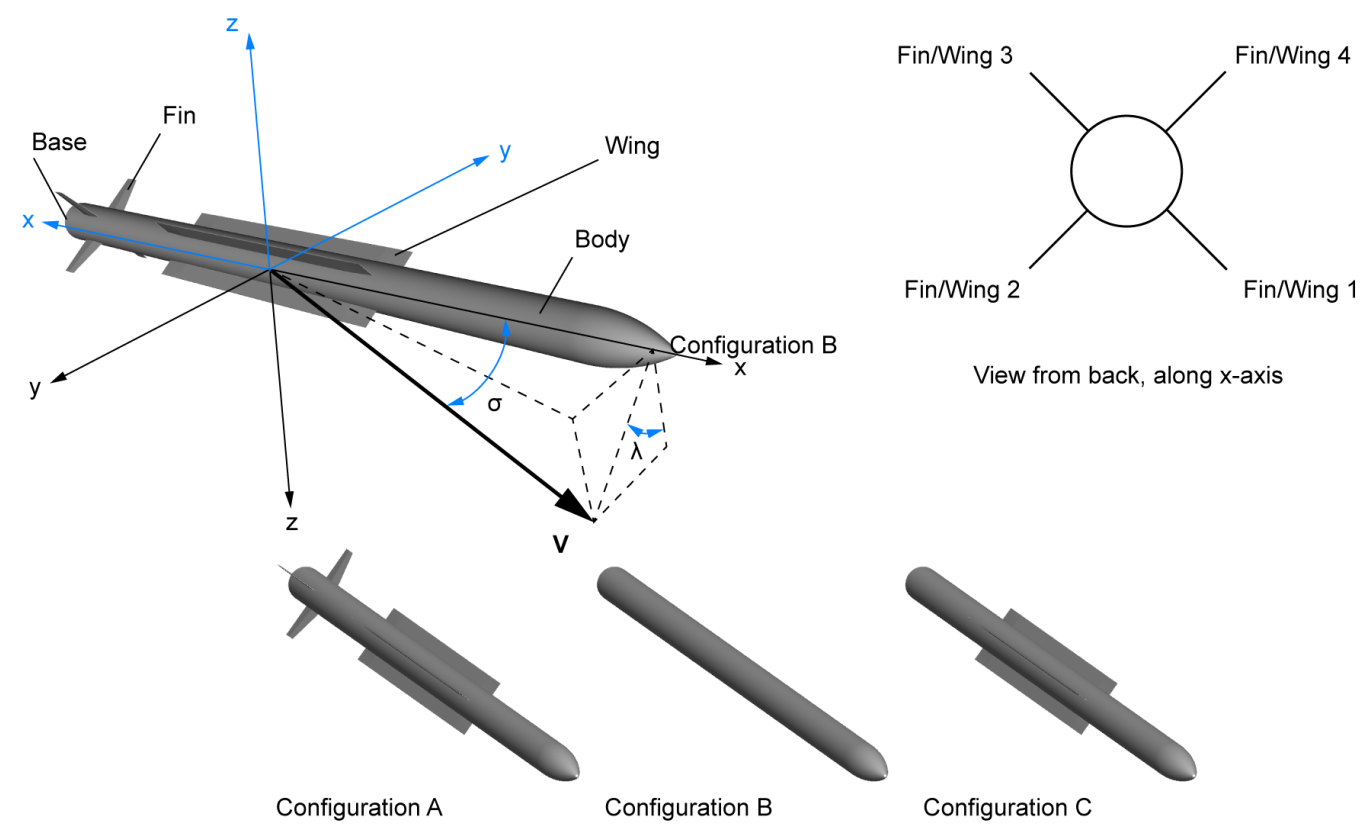

Fig. 2 Geometry of configurations A, B, and C

The right-handed coordinate system is referred to as the body-fixed coordinate system. Rotations and moments are positive clockwise when viewed along the axis of rotation in the positive sense from the origin. The moment reference point is located at $50 \%$ of the total store length. Sea-level (ISA) atmospheric conditions are used. The incidence angle $\sigma$ and the roll angle $\lambda$ are $15.0 \mathrm{deg}$ and $2.5 \mathrm{deg}$ respectively. The Mach number is 1.4 and the Reynolds number based on the store length is $\operatorname{Re}_{L_{\text {ref }}}=112.47 \times 10^{6}$. The velocity components for different $\sigma$ and $\lambda$ values are listed below: 


$$
\mathbf{V}_{\text {ref }}=(u, v, w)^{T} V_{\text {ref }}=\left\{\begin{array}{l}
(0.9659,0.2586,0.0113)^{T} V_{\text {ref }} \text { for } \sigma=15 \text { and } \lambda=2.5 \mathrm{deg} \\
(0.9659,0.2588,0)^{T} V_{\text {ref }} \text { for } \sigma=15 \text { and } \lambda=0 \mathrm{deg} \\
(0.9962,0.0872,0)^{T} V_{\text {ref }} \text { for } \sigma=5 \text { and } \lambda=0 \mathrm{deg}
\end{array}\right.
$$

\section{A. Numerical domain and grids}

ICEM Hexa [27] was used to create high-quality multi-block structured grids. Two grids were created for each level of grid refinement - one for the store and one for the domain around the store. The grids were combined with the overset (Chimera) method. All grids exhibited symmetry across the $x-z$ and $x-y$ planes. The blocking topology of the configuration $\mathrm{C}$ grid is shown in figure 4.
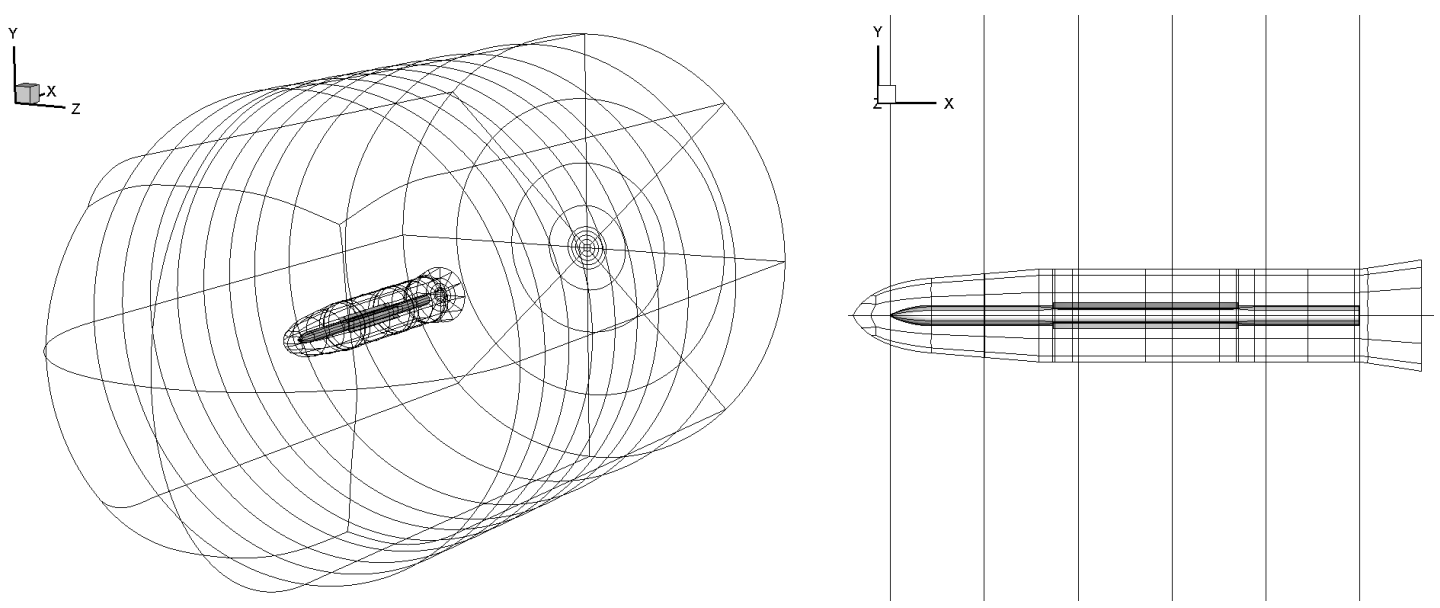

Fig. 3 Multiblock topology used for configuration B.

The grid topology varied slightly for each configuration. Four levels of grid refinement were considered for each configuration. This resulted in 12 store grids and 12 background grids -8 grids per configuration, 24 grids in total. Table 1 lists the grid parameters for all store configurations.

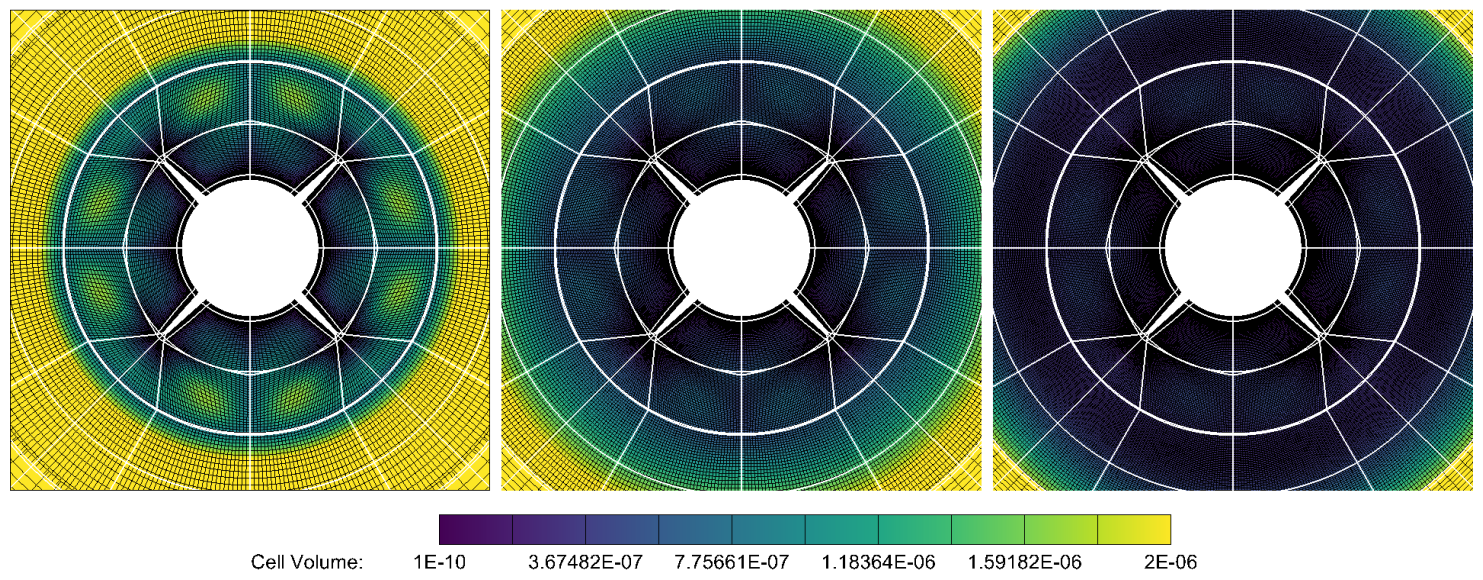

Fig. 4 Configuration B grid slices 
Table 1 Grid parameters; all dimensional quantities are given in millimetres; * as a fraction of $d_{r e f}$.

\begin{tabular}{llcccc}
\hline \hline Grid & Parameter & Fine grid & Very fine grid & Extra-fine grid & Super-fine grid \\
\hline \multirow{4}{*}{ Configuration A } & Domain size $W \times H \times L$ & $80.5 \times 46 \times 46$ & $80.5 \times 46 \times 46$ & $80.5 \times 46 \times 46$ & $80.5 \times 46 \times 46$ \\
& Wake length* & 4.6 & 4.6 & 4.6 & 4.6 \\
& Density radius* & 1.54 & 1.54 & 1.54 & 1.54 \\
& Total cells* $\times 10^{6}$ & 26.8 & 69.2 & 113.5 & 152.3 \\
& Total nodes* $\times 10^{6}$ & 29.9 & 75.0 & 122.0 & 162.5 \\
& First layer height $\times 10^{-3}$ & 3.45 & 3.45 & 3.45 & 3.45 \\
\hline & Domain size* $W \times H \times L$ & $80.5 \times 46 \times 46$ & $80.5 \times 46 \times 46$ & $80.5 \times 46 \times 46$ & $80.5 \times 46 \times 46$ \\
& Wake length* & 3.0 & 3.0 & 3.0 & 3.0 \\
& Density radius* & 1.8333 & 1.83 & 1.83 & 1.83 \\
& Total cells* $\times 10^{6}$ & 43.1 & 80.0 & 127.8 & 190.7 \\
& Total nodes* $\times 10^{6}$ & 47.2 & 86.4 & 136.0 & 201.5 \\
& First layer height $\times 10^{-3}$ & 3.45 & 3.45 & 3.45 & 3.45 \\
\hline & Domain size* $W \times H \times L$ & $80.5 \times 46 \times 46$ & $80.5 \times 46 \times 46$ & $80.5 \times 46 \times 46$ & - \\
& Wake length* & 3.0 & 3.0 & 3.0 & - \\
& Density radius* & 1.83 & 1.83 & 1.83 & - \\
& Total cells* $\times 10^{6}$ & 59.5 & 104.05 & 173.30 & - \\
& Total nodes* $\times 10^{6}$ & 64.8 & 111.76 & 184.07 & - \\
& First layer height $\times 10^{-3}$ & 3.45 & 3.45 & 3.45 & - \\
\hline \hline
\end{tabular}

Figure 4 shows the cell volume at $x / L_{r e f}=0.5$ of the fine, very fine, and extra-fine configuration B grids. The location of the Chimera interface was determined by the position of the vortices generated by the store. For both the $\sigma=5 \mathrm{deg}$ and $\sigma=15 \mathrm{deg}$ incidence angle the vortices created by the store remained within the store attached grid.

Simulations of all configurations were performed with the k- $\omega$ SST turbulence model at $\sigma=15 \mathrm{deg}$. In addition, the effects of the SST, k- $\omega$ EARSM, and k- $\omega$ SST QCR non-linear turbulence models on the predicted flowfield and convergence were investigated. All simulations employed the Osher Riemann solver and used the MUSCL approach to achieve high-order accuracy in space. Solutions were performed implicitly at a CFL of 2.0 for approximately 50000 iterations. All simulations were initialised with free-stream conditions everywhere in the domain. To allow the solution to develop without any numerical instabilities, the solution was obtained explicitly for the first few hundred iterations. Such an approach is not uncommon when initialising supersonic simulations. Simulations with non-linear models were initialised with the results from the k- $\omega$ SST simulations and were run to convergence for an additional 70k iterations. The unsteady SAS simulations were also initialised with the results from the steady k- $\omega$ SST simulations. Table 2 lists the simulation parameters and the obtained force and moment coefficients. 
Table 2 Simulation parameters and obtained force and moment coefficients.

\begin{tabular}{|c|c|c|c|c|c|c|c|c|c|c|}
\hline Configuration & Grid & $\sigma$ & $\lambda$ & $C_{X}$ & $C_{Y}$ & $C_{Z}$ & $C_{l}$ & $C_{m}$ & $C_{n}$ & Model \\
\hline \multirow{4}{*}{ A } & Fine & 15.0 & 2.5 & -0.9352 & -0.7033 & -6.1863 & -0.0298 & -4.5706 & 2.1727 & \multirow{4}{*}{$\mathrm{k}-\omega \mathrm{SST}$} \\
\hline & Very fine & 15.0 & 2.5 & -0.9220 & -0.7169 & -6.2043 & -0.0203 & -4.8504 & 2.1703 & \\
\hline & Extra-fine & 15.0 & 2.5 & -0.9232 & -0.7340 & -6.1096 & -0.0160 & -4.1133 & 2.3312 & \\
\hline & Super-fine & 15.0 & 2.5 & -0.9169 & -0.7400 & -6.1096 & -0.0146 & -4.1280 & 2.3572 & \\
\hline \multirow{4}{*}{ A } & Fine & 15.0 & 2.5 & -0.8051 & -0.7722 & -5.9478 & 0.3485 & -2.3008 & 2.4724 & \multirow{4}{*}{$\mathrm{k}-\omega$ SST SAS } \\
\hline & Very fine & 15.0 & 2.5 & -0.8319 & -0.7854 & -6.0457 & 0.4094 & -3.2320 & 2.4820 & \\
\hline & Extra-fine & 15.0 & 2.5 & -0.8418 & -0.7843 & -5.9473 & 0.5308 & -2.4383 & 1.9510 & \\
\hline & Super-fine & 15.0 & 2.5 & -0.8493 & -0.7222 & -5.9473 & 0.5308 & -2.4383 & 1.9510 & \\
\hline \multirow{4}{*}{ B } & Fine & 5.0 & 0 & -0.6086 & 0.0003 & -0.3519 & 0.0000 & 2.1209 & 0.0023 & \multirow{4}{*}{$\mathrm{k}-\omega \mathrm{SST}$} \\
\hline & Very fine & 5.0 & 0 & -0.6076 & -0.0000 & -0.3369 & 0.0000 & 2.0864 & -0.0005 & \\
\hline & Extra-fine & 5.0 & 0 & -0.6065 & -0.0000 & -0.3268 & -0.0000 & 2.0784 & -0.0004 & \\
\hline & Super-fine & 5.0 & 0 & -0.6071 & 0.0001 & -0.3244 & -0.0000 & 2.0570 & 0.0001 & \\
\hline \multirow{3}{*}{ B } & Very fine & 15.0 & 0 & -0.6924 & -0.0000 & -1.6731 & 0.0000 & 7.5781 & -0.0004 & \multirow{3}{*}{$\mathrm{k}-\omega \mathrm{SST}$} \\
\hline & Extra-fine & 15.0 & 0 & -0.6896 & -0.0001 & -1.6517 & -0.0000 & 7.5627 & 0.0006 & \\
\hline & Super-fine & 15.0 & 0 & -0.6888 & -0.0008 & -1.6401 & -0.0000 & 7.5152 & -0.0020 & \\
\hline \multirow{3}{*}{ B } & Very fine & 15.0 & 0 & -0.5973 & -0.0002 & -1.7324 & -0.0000 & 7.0560 & -0.0014 & \multirow{3}{*}{ k- $\omega$ EARSM } \\
\hline & Extra-fine & 15.0 & 0 & -0.5957 & 0.0002 & -1.7265 & -0.0000 & 7.0934 & -0.0005 & \\
\hline & Super-fine & 15.0 & 0 & -0.5947 & 0.0005 & -1.7258 & -0.0000 & 7.0925 & -0.0045 & \\
\hline $\mathrm{B}$ & Extra-fine & 15.0 & 0 & -0.6849 & -0.0009 & -1.6986 & -0.0000 & 7.4905 & -0.0027 & k- $\omega$ SST QCR \\
\hline \multirow{3}{*}{$\mathrm{C}$} & Fine & 15.0 & 2.5 & -0.7831 & -0.5884 & -4.9706 & -0.0188 & 8.0600 & 0.9271 & \multirow{3}{*}{$\mathrm{k}-\omega \mathrm{SST}$} \\
\hline & Very fine & 15.0 & 2.5 & -0.7805 & -0.6092 & -4.9826 & -0.0163 & 7.9106 & 1.0714 & \\
\hline & Extra-fine & 15.0 & 2.5 & -0.7770 & -0.6194 & -4.9438 & -0.0113 & 7.9829 & 1.2198 & \\
\hline
\end{tabular}

\section{Results and discussion}

\section{A. Configuration A}

First simulations of configuration A were performed on all grids with the k- $\omega$ SST and SAS turbulence models. Here, only results from the k- $\omega$ SST SAS simulations will be discussed as turbulence model investigations showed that the k- $\omega$ SST simulations result in large values of $\mu_{t} / \mu$ which suppresses the formation of vortices around the missile. Figure 5 shows the stagnation pressure contours $p_{0} / p_{\text {ref }}$ at two streamwise locations $\left(x / d_{r e f}=14\right.$ and $\left.x / d_{r e f}=21.5\right)$ for the very fine, extra-fine, and super-fine grids. The contours qualitatively agree, however, there are noticeable differences in the location of the vortices at $x / d_{\text {ref }}=21.5$. The port and starboard vortices are located closer to the surface of fins 3 and 4 on the super-fine grid. The movement of the vortices was observed to affect the pressure distribution and consequently the loads and the convergence. From the component loads breakdown, it was observed that the largest differences in the component loads come from fins 3 and 4 . This shows that the position of the vortices near fins 3 and 4 and their proximity to the surface of the fins can affect the loads significantly. Noticeable differences in the component loads between grids were also observed for wings 1 and 2 . The differences were caused by differences in the location of the same vortices that interact with fins 3 and 4 . These vortices are created by the leading edges of wings 1 and 2 which are positioned on the windward side of the missile. The variation of the forces for each component between grids was observed to be considerably smaller than the variation of the moments.

\section{Effect of turbulence models}

The k- $\omega$ SST, k- $\omega$ EARSM, and k- $\omega$ SST SAS turbulence models were compared on the fine grid. The stagnation pressure, $p_{0} / p_{\text {ref }}$, and eddy viscosity to molecular viscosity ratio $\mu_{t} / \mu$ at $x / d_{\text {ref }}=21.5$ were compared. Figure 6 shows the comparison of the stagnation pressure obtained with the three models. The stagnation pressure contours are saturated to 0.5 and 1.0 for better visualisation of the vortex cores. The k- $\omega$ SST predicts fewer vortex structures than the k- $\omega$ SST SAS and the k- $\omega$ EARSM models. The vorticial structures are not as pronounced as the ones predicted by 

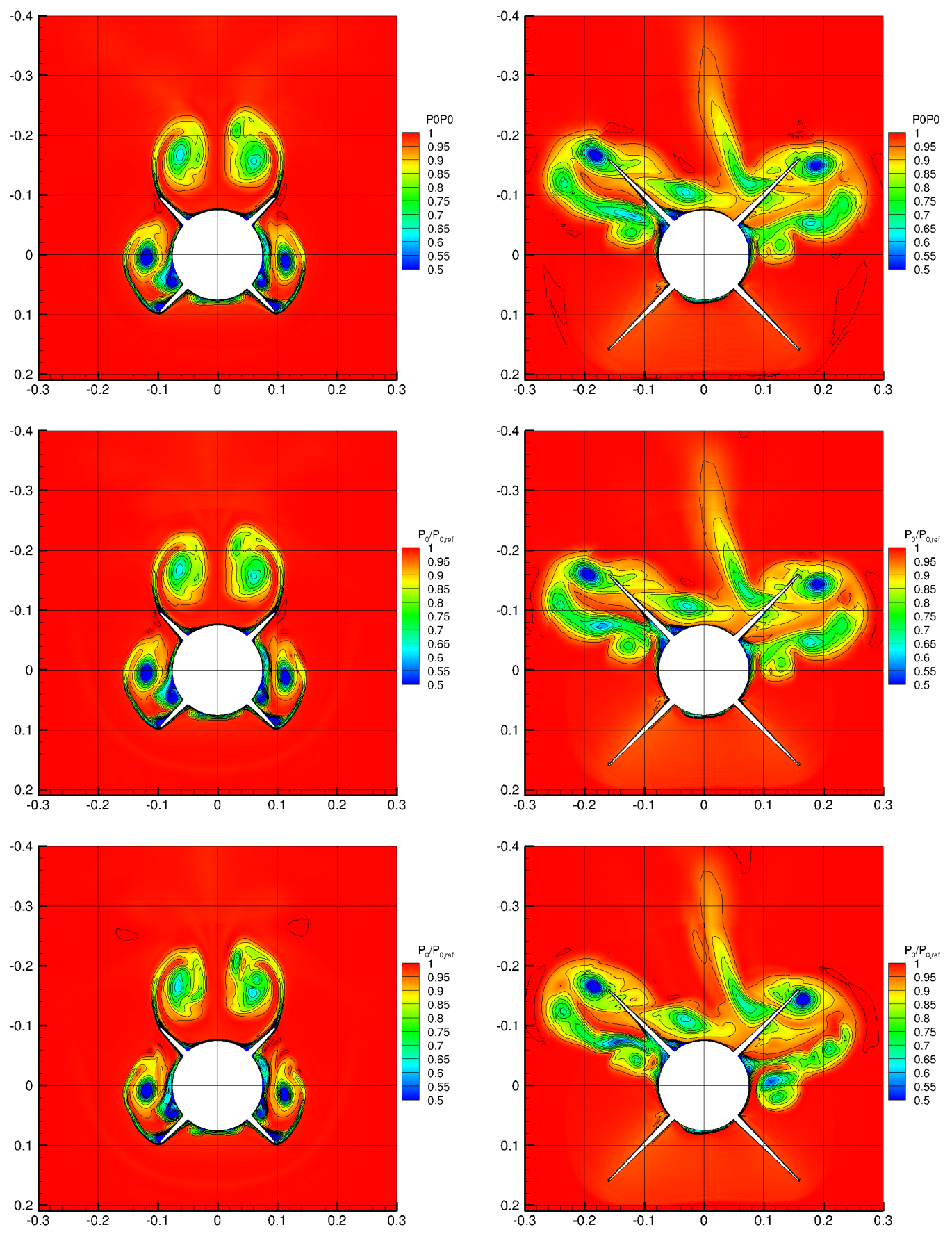

Fig. 5 Stagnation pressure for the k- $\omega$ SST SAS* model on the very fine (top), extra-fine (middle), and super-fine (bottom) grids at $x / d_{\text {ref }}=14$ (left) and $x / d_{\text {ref }}=21.5$ (right); * instantaneous quantities.

the latter turbulence models. Both the k- $\omega$ SAS and the k- $\omega$ EARSM predict two strong vortices at the vicinity of fins 3 and 4 . The presence of these strong vortices affects the pressure distribution over the tail which consequently affects the loads. The rolling moment predicted by the k- $\omega$ SST is very small compared to the one predicted by the k- $\omega$ SAS and k- $\omega$ EARSM models due to the inability of the model to predict these vortices. Figure 7 shows the comparison of the modelled eddy to molecular viscosity ratio (RET). The contours are saturated to 0 and 2000 for better visualisation. At $x / d_{\text {ref }}=21.5$ location the $\mathrm{k}-\omega \mathrm{SST}$ model predicts high values for the $\mu_{t} / \mu$ ratio. 

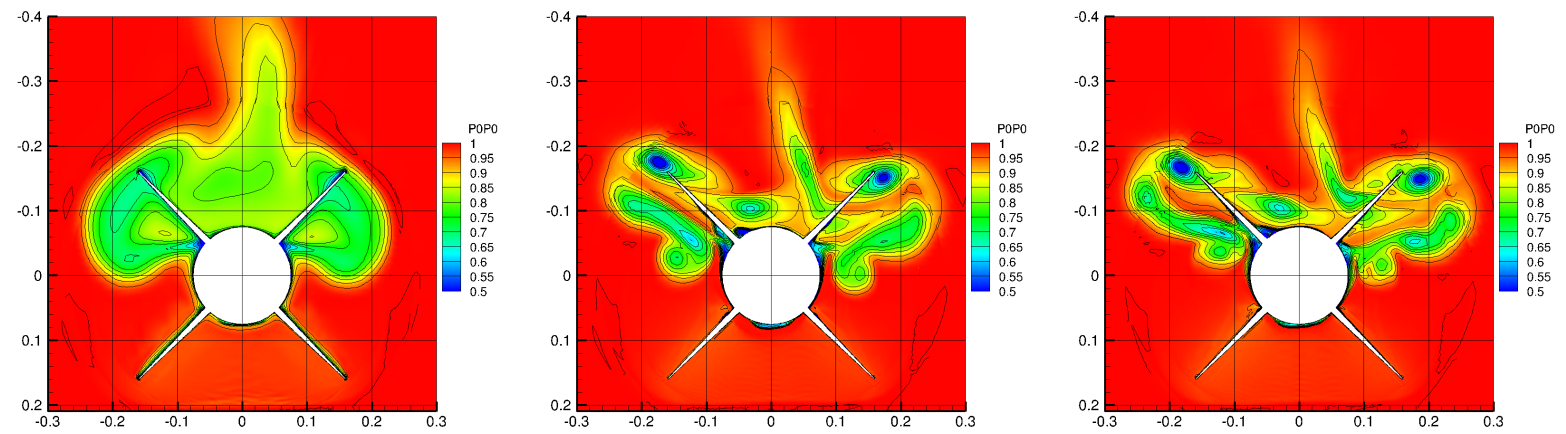

Fig. 6 Stagnation pressure for the k- $\omega$ SST (left), the k- $\omega$ EARSM (middle), and the k- $\omega$ SST SAS* (right) models on the very fine grid at $x / d_{r e f}=21.5$; $^{*}$ - instantaneous quantities.

High values of $\mu_{t} / \mu$ prevent the development of instabilities in the flow, hence less vorticial structures are observed in the k- $\omega$ SST solution. The modelled turbulent eddy viscosity $\mu_{t}$ is reduced in the k- $\omega$ SAS simulations due to the increase of the $Q_{S A S}$ term in the $\omega$-equation. Due to the lower eddy viscosity flow structures can develop and this can result in a complex flowfield. The k- $\omega$ EARSM model also predicts lower values for $\mu_{t}$ which results in more vorticial structures in agreement with the k- $\omega$ SAS solution.
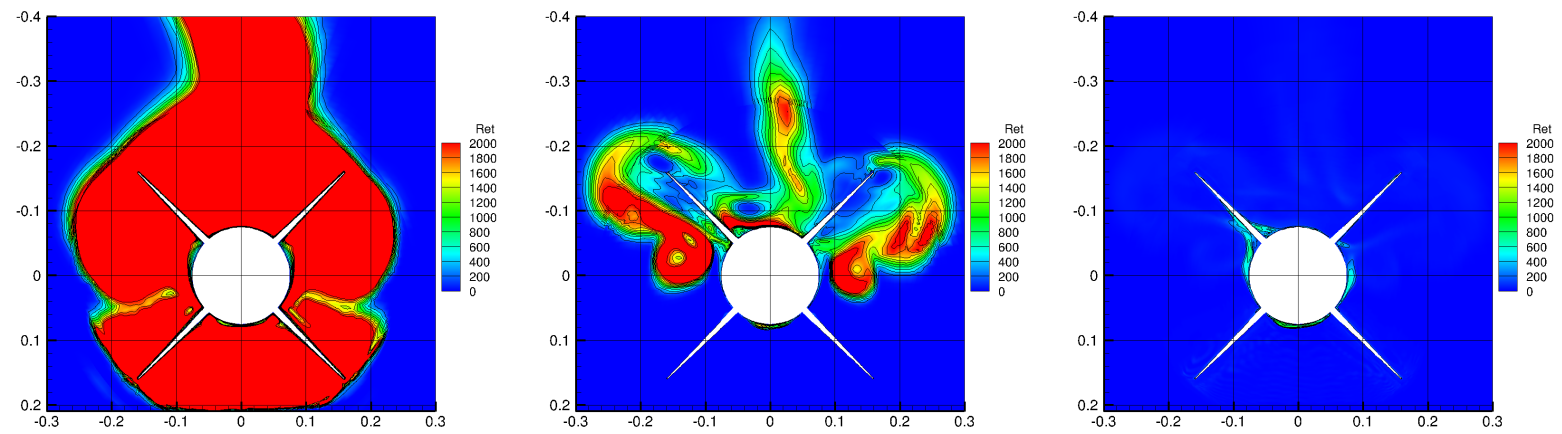

Fig. 7 RET for the k- $\omega$ SST (left), the k- $\omega$ EARSM (middle), and the k- $\omega$ SST SAS* (right) models on the very fine grid at $x / d_{\text {ref }}=21.5$; $^{*}$ - instantaneous quantities.

Figure 8 compares the trajectory of the wing 1 vortex for the k- $\omega$ SST, k- $\omega$ EARSM, and the k- $\omega$ SST SAS models. The vortex trajectories were obtained with the $\lambda_{2}$ method. The dependency of the vortex trajectory on the turbulence model is clearly visible. Both the k- $\omega$ EARSM and the $\mathrm{k}-\omega$ SST SAS models predict the wing 1 vortex to pass below fin 4. In addition, the difference between the k- $\omega$ EARSM and the k- $\omega$ SST SAS vortex trajectories in the vicinity of fin 4 contributes to the difficulty in obtaining grid converged results. 


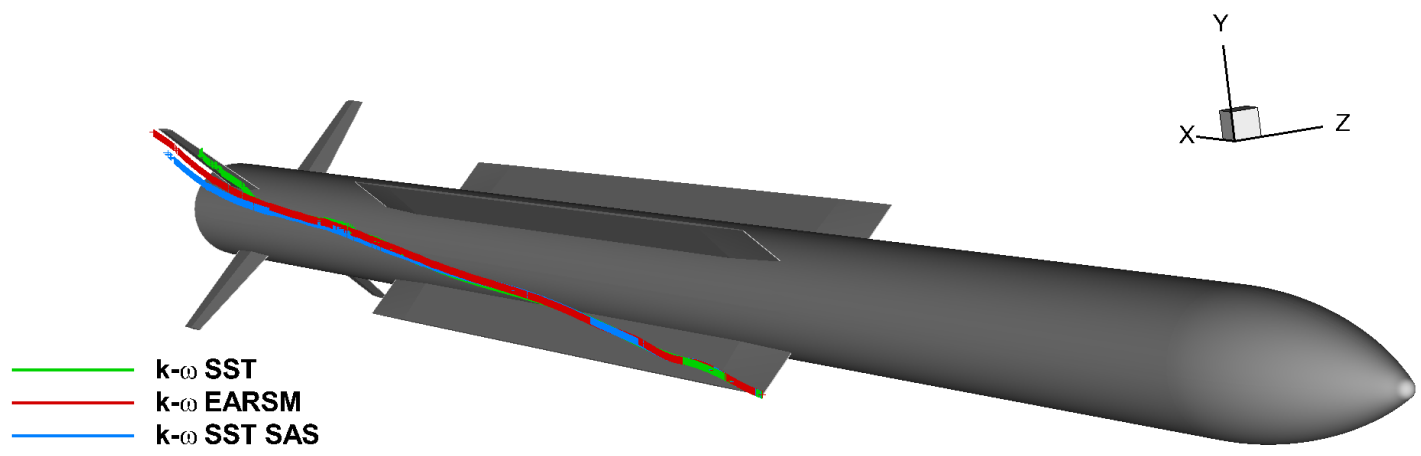

Fig. 8 Trajectory of wing 1 vortex for the k- $\omega$ SST (left), the k- $\omega$ EARSM (middle), and the k- $\omega$ SST SAS (right) models on the very fine grid.

\section{B. Configuration B}

To investigate the the lack of mesh convergence of configuration A, simulations of configuration B at an incidence angle of $\sigma=5 \mathrm{deg}$ were performed. Figure 9 shows the iteration history of the aerodynamic loads. The large difference in $C_{Z}$ between the fine, very fine, and extra-fine grid shows that grids of the same size as the very fine grid or larger must be used to accurately predict $C_{Z}$. A further increase of the grid size by $65.32 \times 10^{6}$ points reduced the difference in $C_{Z}$ significantly. The $C_{X}, C_{Z}$, and $C_{m}$ aerodynamic coefficients were observed to settle after approximately 30000 iterations and exhibited monotonicity i.e. all values were increasing or decreasing as the grid was refined. A total of 50k iterations were performed for the $C_{X}, C_{Z}$, and $C_{m}$ to show iterative convergence.
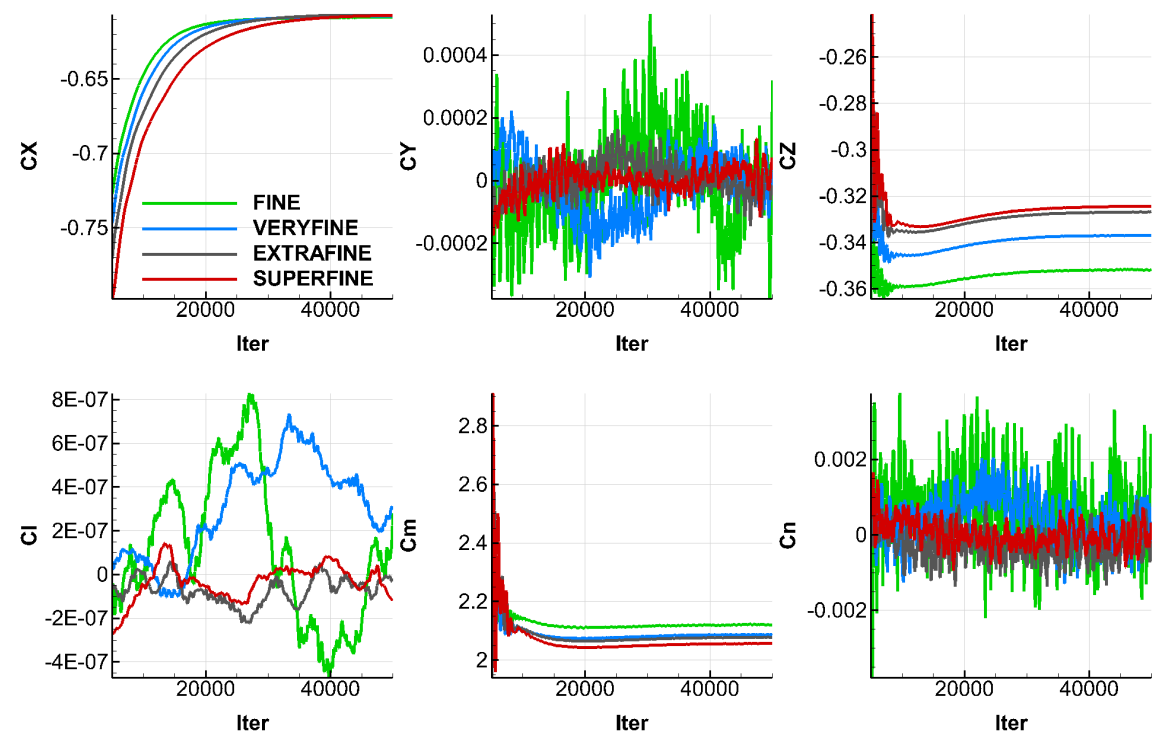

Fig. 9 Aerodynamic loads for configuration B resulting from pressure and viscous contributions at $\sigma=5 \mathrm{deg}$

The viscous contributions to the $C_{m}$ and $C_{Z}$ aerodynamic loads exhibited a non-monotonic behaviour. The value of $C_{m}$ on the very fine grid was less than the value on the extra-fine grid, which was greater than the value on the super-fine grid. Even though the viscous values for $C_{Z}$ and $C_{m}$ were non-monotonic the total values were, due to the small contribution of the viscous values. The difference in the total $C_{m}$ value between the super-fine and extra-fine grid was greater than between the extra-fine and very fine grids. One would expect the difference in all aerodynamic coefficients to decrease as the grid is refined ( $C_{Z}$ is such an example). Further analysis of the $C_{m}$ values per component (store body and base) and per contribution (pressure and viscous) showed that the difference in $C_{m}$ originates from the store body. Figure 10 shows the stagnation pressure contours $p_{0} / p_{\text {ref }}$ and the TKE contours $k / V_{r e f}^{2}$ at the $x / d_{r e f}=21.5$ 
streamwise location (base). Only the very fine, extra-fine, and super-fine clean grids are shown. The fine grid is omitted due to the large difference in the predicted $C_{Z}$ value.
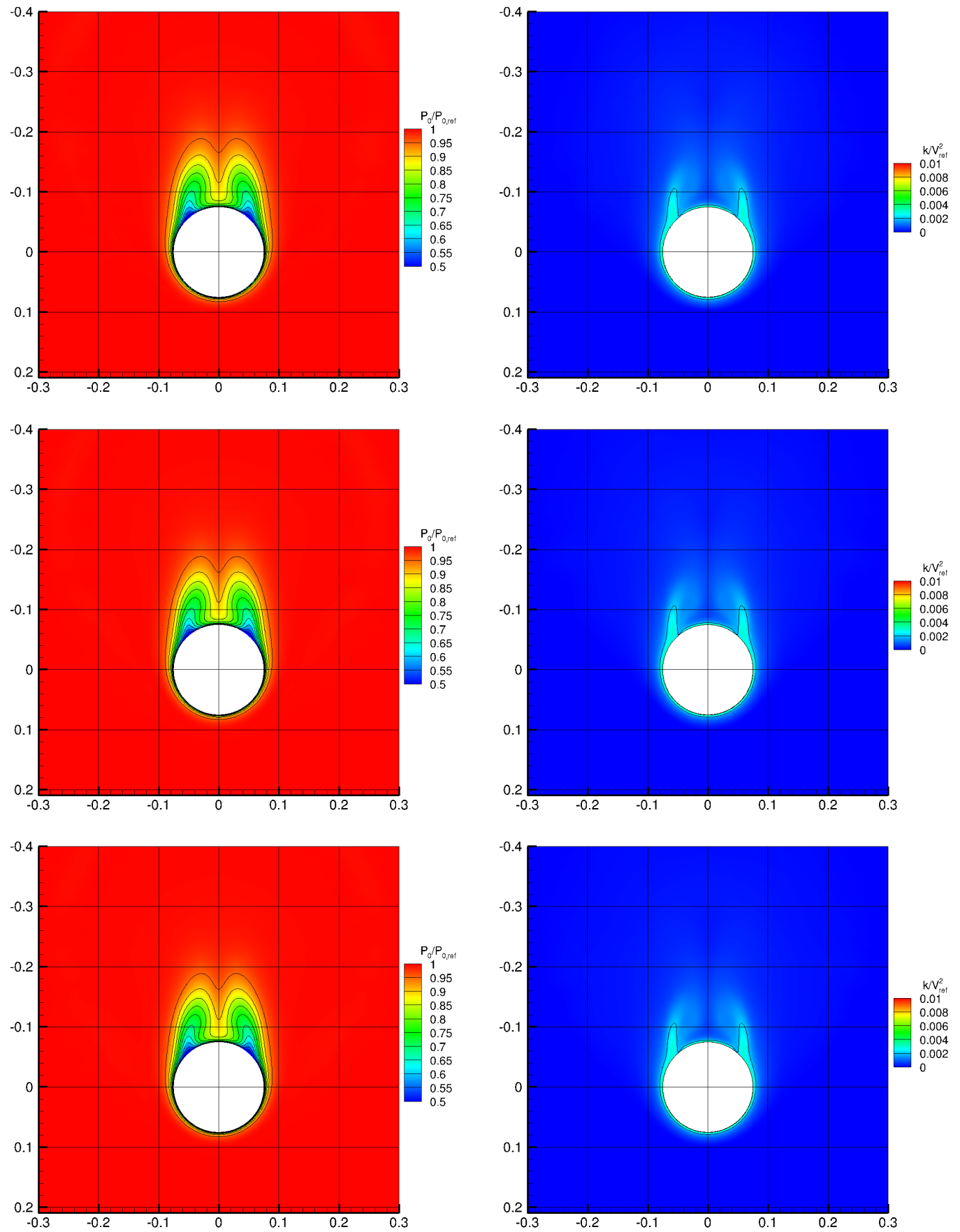

Fig. 10 Stagnation pressure (left) and TKE (right) for the k- $\omega$ SST model on the very fine (top), extra-fine (middle), and super-fine (bottom) grids at $x / d_{\text {ref }}=21.5$.

No significant differences in the stagnation pressure and TKE contours were observed between the three grids. It is, therefore, hard to identify the cause for the differences in the aerodynamic loads solely from the stagnation or TKE contour plots. A better comparison is shown in figure 11. The figure shows the difference in pressure coefficient between the four grids. 


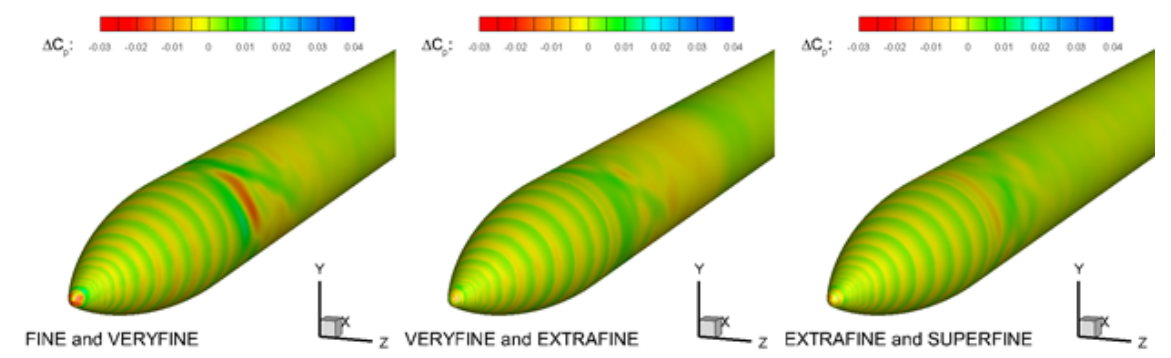

Fig. 11 Difference in pressure coefficient $\Delta C_{p}$ between the fine and very fine (left), the very fine and extra-fine (middle) and extra-fine and super-fine (right).

The largest $\Delta C_{p}$ was observed near the nose of the store, downstream differences in $C_{p}$ were insignificant. As the grid was refined, $\Delta C_{p}$ reduced, however, it was still identifiable. In addition, pressure contours between the three grids were compared directly by overlaying them on top of each other. In the area of the nose, the pressure contours showed good agreement between the three grids. Downstream the nose, however, noticeable differences were observed. The low-pressure region on the leeward side of the store body, extending to the port and starboard sides changed its shape slightly as the grid was refined. A change in the pressure region on the windward side was also observed. Since the pressure distribution on the windward and leeward sides is the main contributor to the pitching moment, differences in the pressure distribution in these areas between grids are expected to affect the pitching moment. The pressure contours on the base showed no significant differences, further supporting the component analysis which showed that the base is not contributing to the differences in $C_{m}$. Comparing the windward and leeward streamwise $C_{p}$ distribution showed small changes in $C_{p}$ as the grid was refined. At $x / L_{r e f}=-0.4$ the super-fine grid gave a slightly higher $C_{p}$ than the extra-fine grid. This difference may be the cause for the difference in the pitching moment coefficient $C_{m}$ between the extra-fine and super-fine grids.

\section{Configuration $B$ at high incidence angle}

Similar observations for configuration B were made at $\sigma=15 \mathrm{deg}$ incidence angle. The aerodynamic loads exhibited monotonic behaviour. The change in the pitching moment coefficient between the extra-fine and super-fine grids was again slightly larger than the change between the very fine and extra-fine grids. The surface pressure contours and the vortex trajectories agreed well between the grids. Figure 12 shows the stagnation pressure $p_{0} / p_{\text {ref }}$ and the turbulent kinetic energy $k / V_{\text {ref }}^{2}$ contours at the $x / d_{\text {ref }}=21.5$ streamwise location (the store base).

\section{Non-linear models on configuration $B$}

The effect of the k- $\omega$ EARSM and the k- $\omega$ SST QCR V1 models on the loads was investigated. The k- $\omega$ EARSM simulation was restarted from the k- $\omega$ SST simulation and run for additional 70000 iterations. A large number of iterations was necessary for the loads to settle. The largest difference was in the $C_{X}$ coefficient - approximately $13.4 \%$ relative to the k- $\omega$ SST simulation. The differences in $C_{Z}$ and $C_{m}$ were approximately $4.6 \%$ and $6.3 \%$. Figure 13 shows a side-by-side comparison of the stagnation pressure contours predicted by the k- $\omega$ SST and k- $\omega$ EARSM models. The linear k- $\omega$ SST model predicts higher values for $\mu_{t} / \mu$ which reduces unsteadiness in the flow and results in fewer vortical structures. The k- $\omega$ EARSM model, which considers higher-order strain and rotation terms, reduces the $\mu_{t} / \mu$ values and results in more pronounced vortical structures with more laminar cores. Unlike the k- $\omega$ SST model, the values of $k / V_{\text {ref }}^{2}$ at the vortex cores are small. The k- $\omega$ SST QCR V1 was tested only on the extra-fine grid. No significant differences in the loads or in the flow topology were observed when compared to the k- $\omega$ SST. Differences between the two models are expected to occur with the inclusion of wings and/or fins as the right angles between the fins and/or wings and the body give rise to secondary flows which will be accounted for by the k- $\omega$ SST QCR V1 model only.

\section{Unsteady simulations of configuration B}

Unsteady RANS simulations of the body at $\sigma=15$ degrees were also performed to identify unsteadiness if any. A non-dimensional time step of $\Delta t=0.01$ was used which corresponded to a dimensional time step of $7.24 \times 10^{-5}$ $\mathrm{s}$ based on $L_{r e f}$ and $3.15 \times 10^{-6} \mathrm{~s}$ based on $d_{r e f}$. A total of 6 flow travel times were simulated where 1 flow travel 

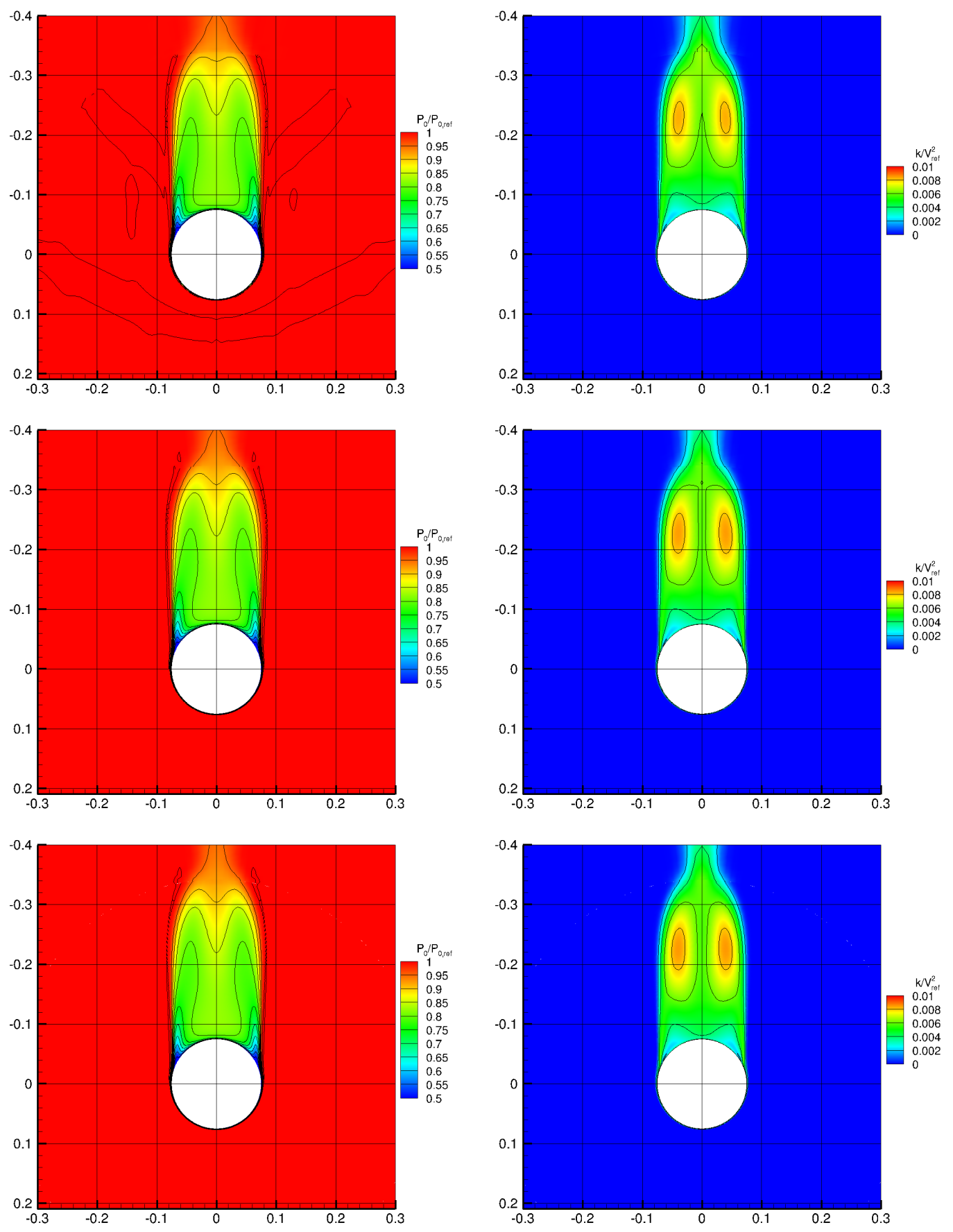

Fig. 12 Stagnation pressure (left) and TKE (right) for the k- $\omega$ SST model on the very fine (top), extra-fine (middle), and super-fine (bottom) grids at $x / d_{\text {ref }}=21.5$.

time was the time it took the flow to travel the length of the body. Averaging was performed over different intervals, however, no significant differences in the aerodynamic coefficients were observed as long as the first travel time was discarded during the averaging. No significant differences in the aerodynamic coefficients were observed. Table 3 lists the averaged aerodynamic coefficients. 

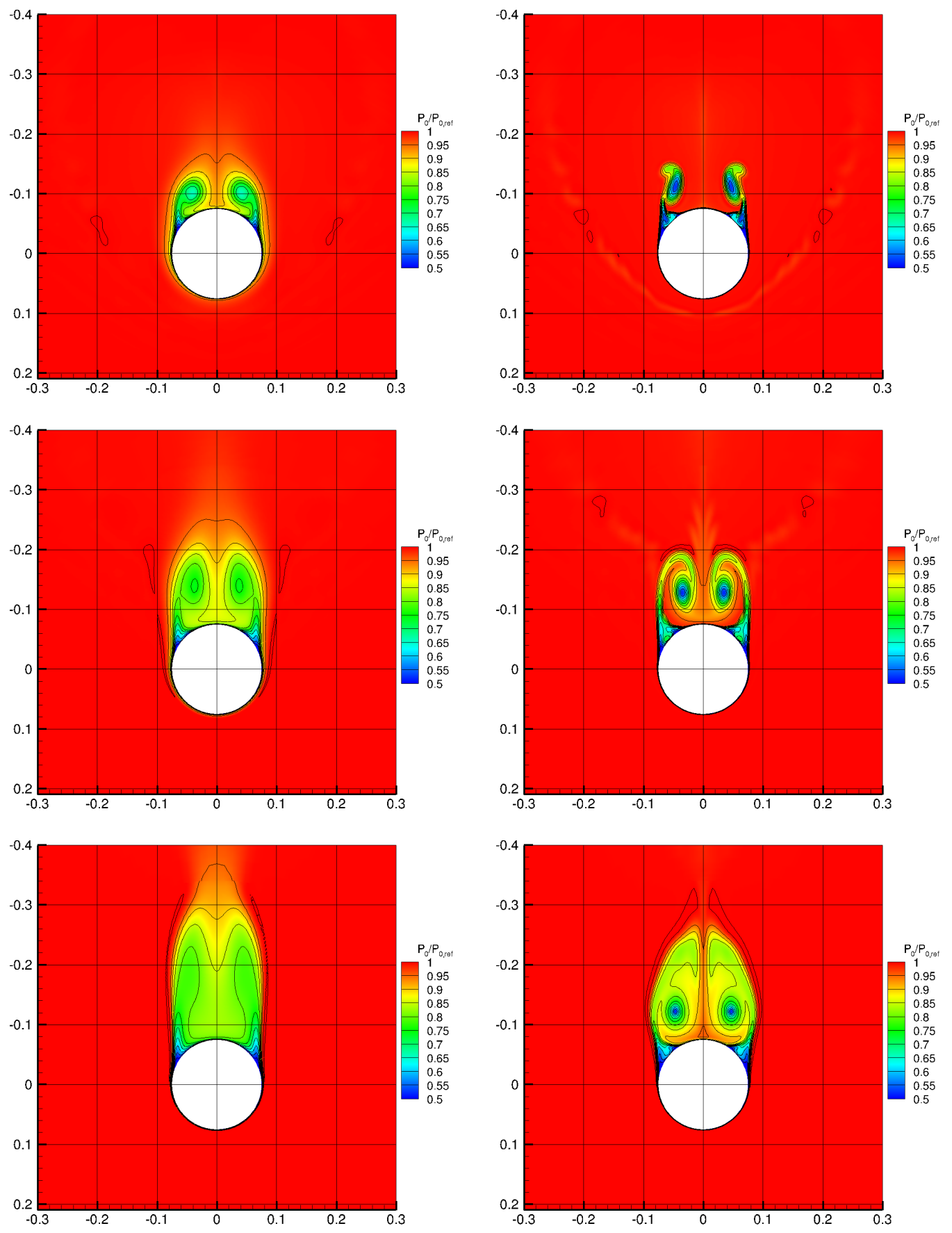

Fig. 13 Stagnation pressure contours for the $\mathrm{k}-\omega \mathrm{SST}$ model (left) and the k- $\omega$ SST model (right) at $x / d_{\text {ref }}=7.5$, $x / d_{\text {ref }}=12.5$, and $x / d_{\text {ref }}=19$. 


\section{Configuration $\mathbf{C}$}

Figure 14 shows the iteration history of the aerodynamic loads. The total loads, including pressure and viscous contributions, and the pressure and viscous only loads were considered. For all grids the loads begin to stabilise after the 30000th iteration, however, results are compared at the final - 50000th iteration. All of the loads apart from $C_{Z}$ and $C_{m}$ exhibit monotonic behaviour. The largest difference is observed in the $C_{Z}$ aerodynamic coefficient. By examining the pressure and viscous loads separately it was observed that the non-monotonicity originates from the pressure loads.
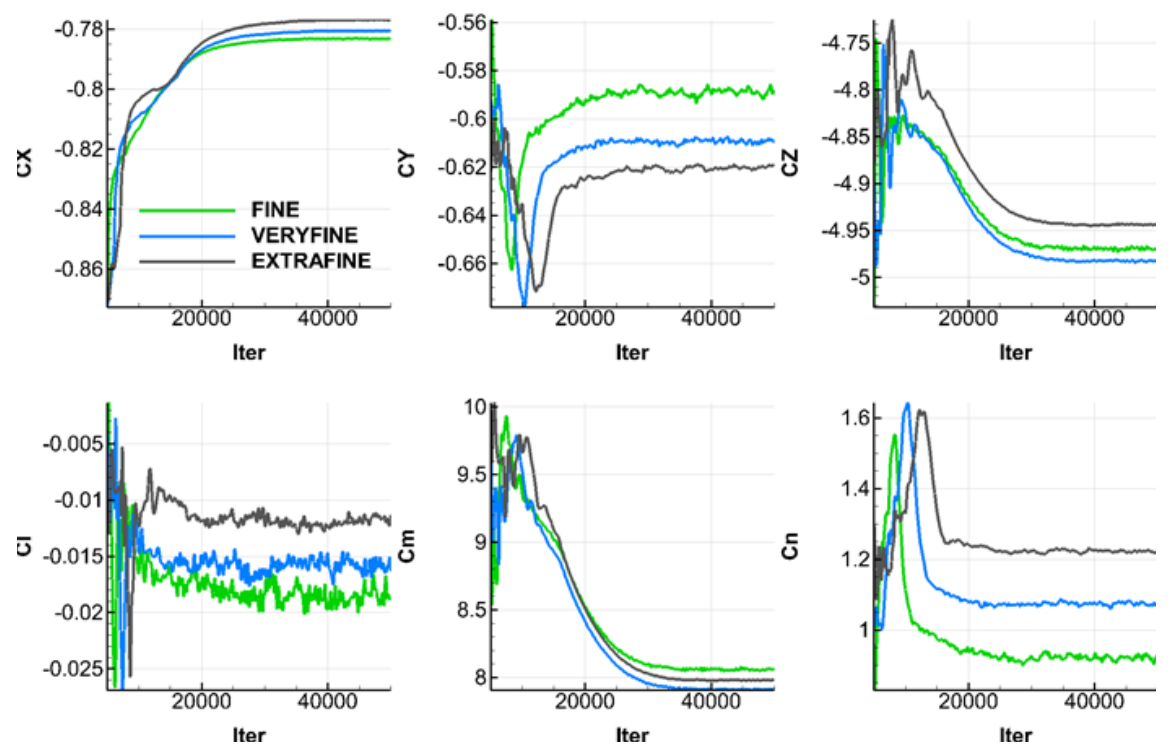

Fig. 14 Aerodynamic loads for configuration $\mathbf{C}$ resulting from pressure and viscous contributions at $\sigma=15$ deg

To further investigate the origin of the non-monotonicity in $C_{Z}$ and $C_{m}$ caused by the pressure loads, figure 15 shows the stagnation pressure $p_{0} / p_{\text {ref }}$ and the TKE contours $k / V_{\text {ref }}^{2}$ at the $x / d_{\text {ref }}=12.5$ streamwise location. The cores of the vortices generated by the windward wings were stronger on the very fine and extra-fine grids, however, no significant differences in the vortex topology from the $p_{0} / p_{\text {ref }}$ contours were observed between the grids. Similarly, the TKE contours show little difference between the three grids. It is generally hard to determine the origin of the differences in the aerodynamic coefficients from the stagnation or TKE contour plots alone. A better comparison is shown in figure 16 The figure compares the difference in pressure coefficient between the three grids. Red corresponds to areas of large negative change and blue corresponds to areas of large positive change in $C_{p}$. Such comparison often allows the origin of the differences in the aerodynamic coefficients to be determined. Large differences in $C_{p}$ between the grids were observed near the leading edges and between the wings. Refining the grid reduced the differences, however, the locations of the largest differences remained the same. Since the difference in $C_{p}$ between the grids was obtained with an interpolation method, the method itself might have introduced additional unwanted uncertainty. To reduce the uncertainty, the surface pressure contours were compared between the three grids directly. The pressure contours agreed well between grids. Only slight differences were observed in the extra-fine grid. To further investigate the origin of the differences in $C_{Z}$ and $C_{m}$ between the grids, the streamwise distribution of the aerodynamic coefficients was considered. The distribution was obtained by taking approximately 225 equally spaced slices. The aerodynamic coefficients for each slice were obtained from the pressure distribution by integration. Figure 17 shows the streamwise variation of the aerodynamic coefficients. No significant differences were observed in the streamwise variation of the $C_{X}, C_{m}, C_{Z}$ aerodynamic coefficients. More significant differences were observed in the $C_{Y}$ and $C_{n}$ aerodynamic coefficients at $x / L_{\text {ref }}=0.9$. There was also a significant difference in the $C_{l}$ aerodynamic coefficient at $x / L_{\text {ref }}=0.61$. The difference in $C_{Z}$ and $C_{m}$ observed previously in figure 14 was not present in the streamwise variation of $C_{Z}$ and $C_{m}$. Due to the equal spacing of the slices, the resolution near the leading edge of the wings is low. Larger differences in $C_{Z}$ and $C_{m}$ may be observed in this area if the number of slices (resolution) is increased. The $\lambda_{2}$ method was used to identify the vortex cores. Overall the locations of the vortex cores agreed well between the three grids. 

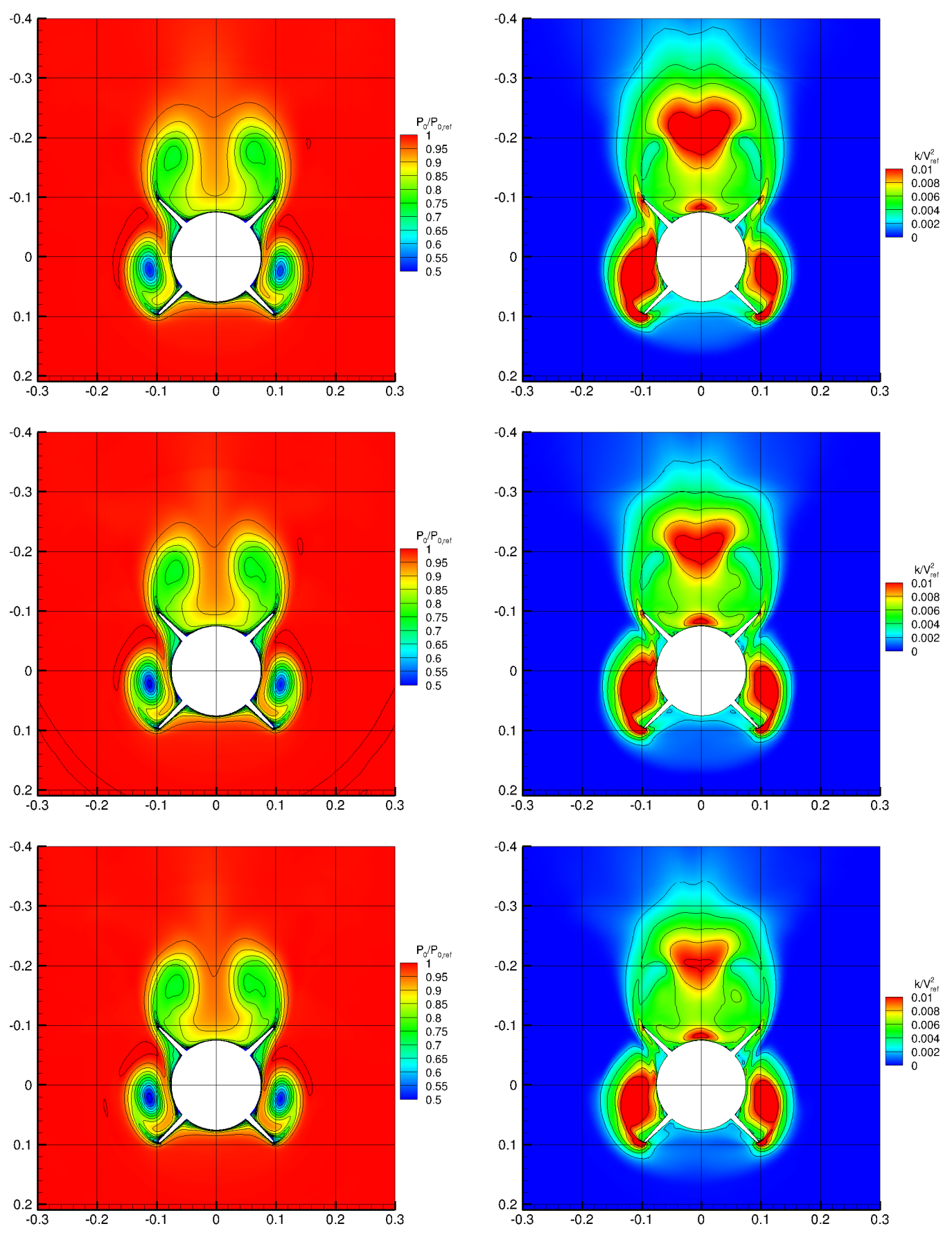

Fig. 15 Stagnation pressure (left) and TKE (right) for the k- $\omega$ SST model on the fine (top), very fine (middle), and extra-fine (bottom) grids at $x / d_{r e f}=21.5$.

Slight differences were observed in the location where the vortices created from the leeward wings interacted with the vortices created from the body. Figure 18 shows the stagnation pressure contour slices along the store. Values of $p_{0}$ greater than 0.9 are clipped. 


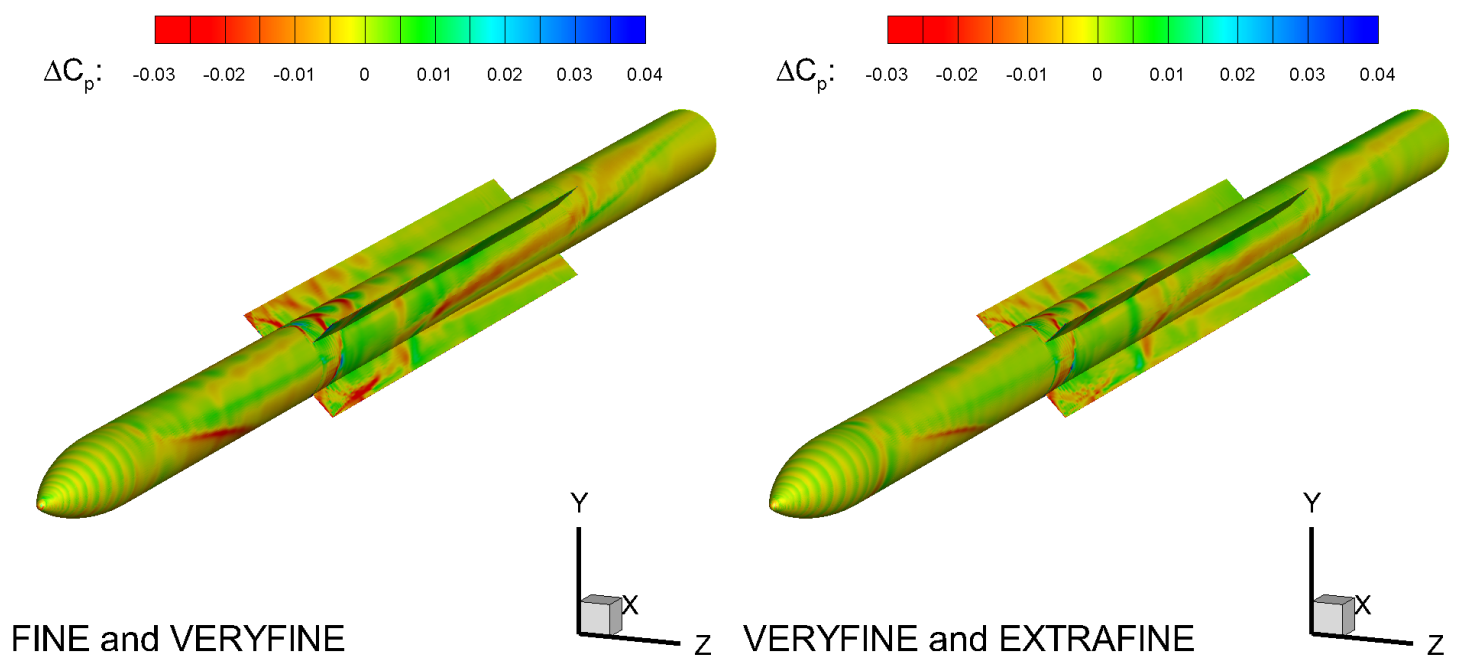

Fig. 16 Difference in pressure coefficient $\Delta C_{p}$ between the fine and very fine (left), the very fine and extra-fine (right) grids.
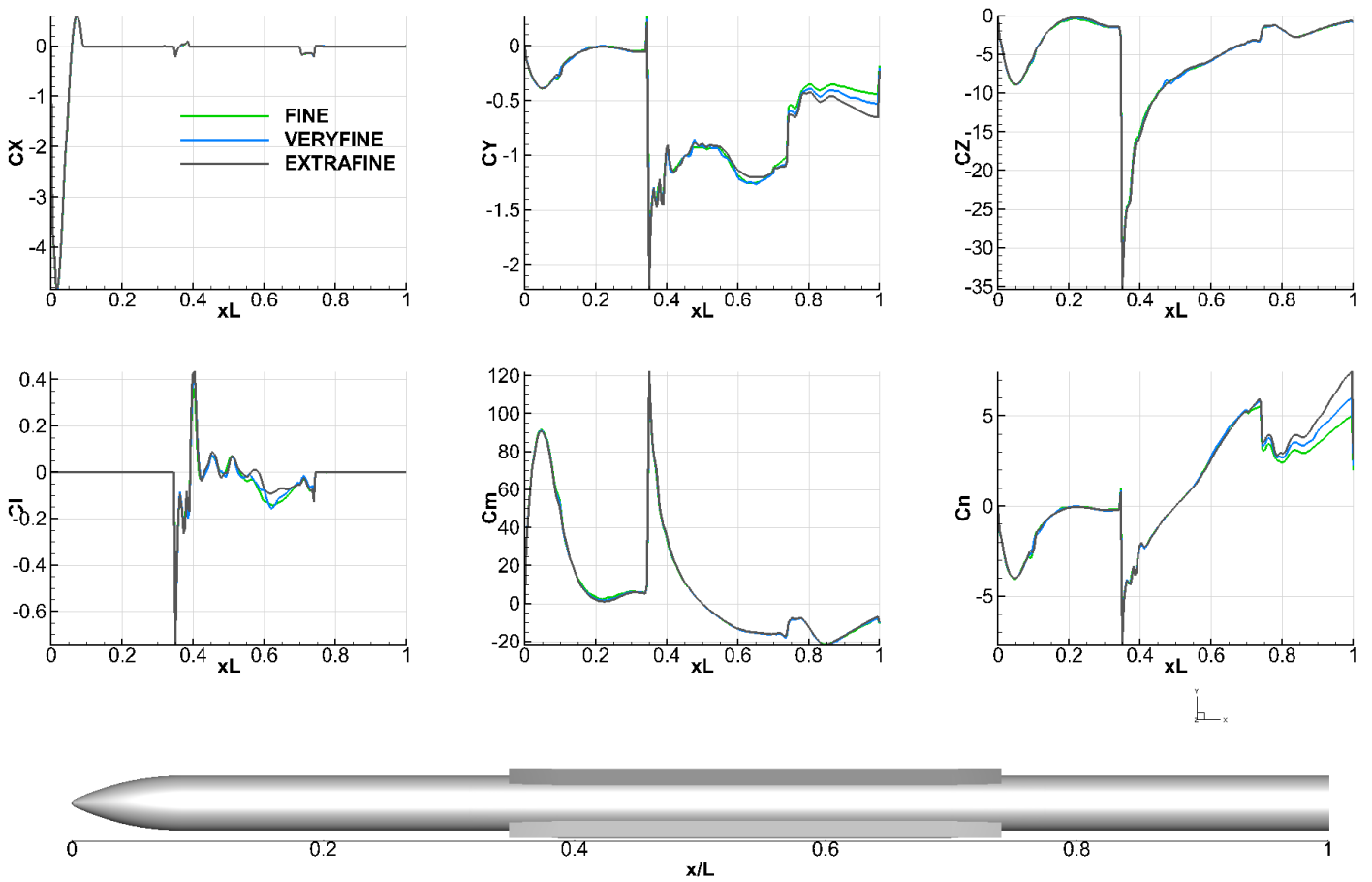

Fig. 17 Streamwise variation of the aerodynamic coefficients for the k- $\omega$ SST model on the fine, very fine, and extra-fine grids. 


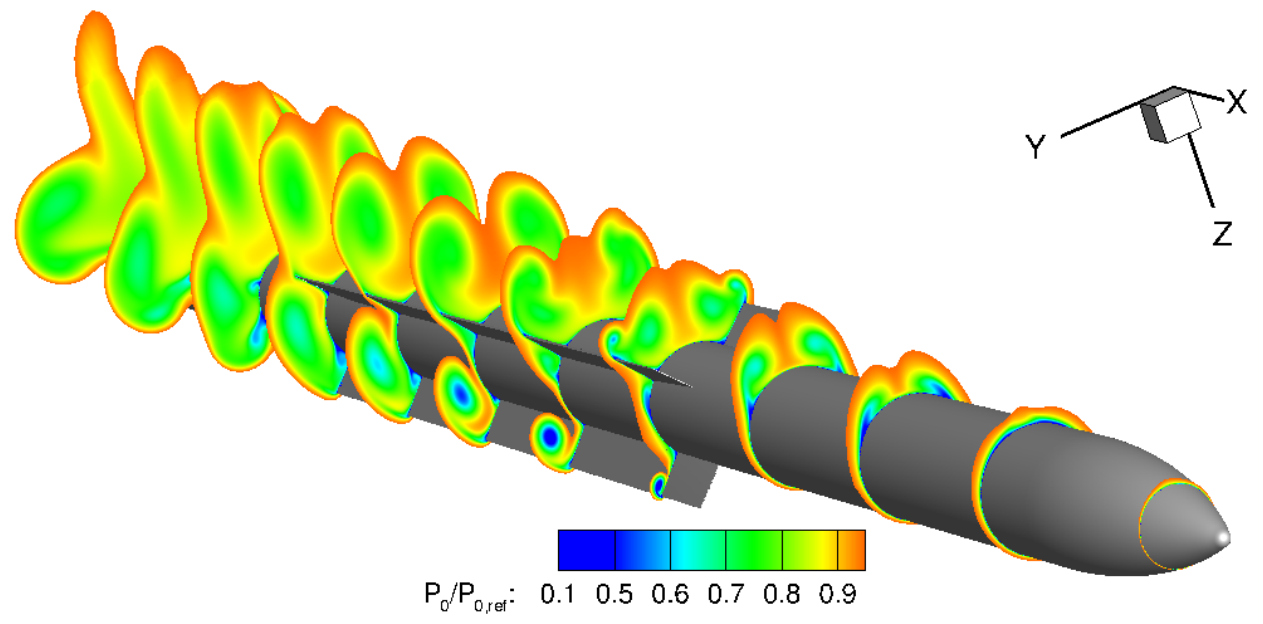

Fig. 18 Stagnation pressure contours for the k- $\omega$ SST model on the extra-fine grid.

Table 3 Unsteady simulations parameters; $t$ is the time it takes for the flow to travel one store body length

\begin{tabular}{lccc}
\hline \hline$C_{X}$ & $C_{Z}$ & $C_{m}$ & Averaging interval \\
\hline-0.690 & -1.651 & 7.574 & $2 \mathrm{t}-6 \mathrm{t}$ \\
-0.690 & -1.651 & 7.574 & $3 \mathrm{t}-6 \mathrm{t}$ \\
-0.690 & -1.651 & 7.574 & $4 \mathrm{t}-6 \mathrm{t}$ \\
-0.690 & -1.651 & 7.574 & $5 \mathrm{t}-6 \mathrm{t}$ \\
\hline-0.690 & -1.652 & 7.563 & RANS \\
\hline \hline
\end{tabular}




\section{Conclusions}

The flow around a generic store geometry at a high angle of incidence was investigated. The flow features vortices that originate from the fore-body and windward wings of the store and interact with the downstream fins on the store. Predicting the interaction of these vortices with the downstream fins of the store is key to predicting accurately the aerodynamic loads acting on the store. The k- $\omega$ SST SAS simulation showed that grid convergence is difficult to obtain due to changes in the location of the vortices with respect to the store fins. Even if the location change is not very significant, the aerodynamic forces and moments of the fin are affected. The forces of each component were less sensitive to changes in the location of the vortices than the moments. Comparison of the different turbulence models and methods (statistical and scale-resolving) showed that the k- $\omega$ SST model predicts higher levels of eddy viscosity which inhibits the formation of instabilities in the flow and results in less vorticial structures which leads to considerably smaller rolling moments. The k- $\omega$ EARSM model predicted significantly smaller values of eddy viscosity and the predicted location and size of the vortices compared favourably with the scale-resolving k- $\omega$ SST SAS method. As the k- $\omega$ EARSM model and the k- $\omega$ SST SAS method reduced the eddy viscosity, which improved the prediction of the aerodynamic loads, especially of the rolling moment, they are put forward as a suitable method for simulating the vortical flows around store configurations. The non-linear k- $\omega$ EARSM can offer a significant reduction in computational resources compared to a scale resolving method such as the k- $\omega$ SST SAS. The favourable agreement between the statistical non-linear model and the scale resolving method showed that the prediction of the loads is not driven by an unsteadiness, but from the position of the vortices with respect to the fins. Additional simulations of simplified store geometries showed that statistical models can be used to obtain grid converged results. Some of the predicted aerodynamic loads for configurations $\mathrm{B}$ and $\mathrm{C}$ exhibited monotonic behaviour. For the latter, the differences between the aerodynamic loads were larger, compared to the geometry without wings or fins. The improved convergence for the simplified geometries showed that the difficulty in obtaining grid converged results for configuration A comes from the interaction of the wing 1 vortex with fin 4 and from the interaction of the body and wing vortices. The location of the vortices past the interaction or their merging location needs to be resolved better. Further work will focus exclusively on RANS and scale-resolving simulations of the store body with wings. Detailed investigation of the iterative convergence and the integrated loads will be performed component by component to further support that the lack of convergence for the full store is caused by the complex interaction between the vortices and the fins. This work is carried out as a part of the NATO AVT-316 activity [1, 26].

\section{Acknowledgments}

The author (K.Boychev) would like to acknowledge the support from MBDA UK throughout his PhD study. The work in this paper was carried out as a part of the $\mathrm{PhD}$ study.

In addition, the authors would like to acknowledge the contributions made to this paper by their fellow AVT 316 Missile Faceteers: (in alphabetical order, by organisation) Michael Anderson, Kilian Cooley \& Greg McGowan (Corvid Technologies), Christian Schnepf (German Aerospace Center, DLR), Magnus Tormalm (Swedish Defence Research Agency, FOI), Henry Bucklow (ITI), Gaëtan. Loupy (MBDA F), Scott Shaw and Nigel Taylor (MBDA UK), Michael A. Park (NASA Langley Research Center), Erdem Dikbaş (TÜBİTAK SAGE) and James DeSpirito (U.S. Army Research

Laboratory). As befits a missile-related team, there have been no passengers in the pursuit of our objectives and our shared experience has provided a valuable backdrop for this paper.

\section{References}

[1] Taylor, N., et al., "The Prediction of Vortex Interactions on a Generic Missile Configuration Using CFD: Current Status of Activity in NATO AVT-316," NATO STO-MP-AVT-307, 2019.

[2] Taylor, N., "AVT-316 Missile Facet: Overview of its Formation, Objectives and Manner of Working," Paper to be presented at AIAA SciTech 2022, 2022.

[3] Dikbaş, E., et al., "The Influence of the Computational Mesh on the Prediction of Vortex Interactions about a Generic Missile Airframe," Paper to be presented at AIAA SciTech 2022, 2022.

[4] Park, M., and DeSpirito, J., "The Influence of Adaptive Mesh Refinement on the Prediction of Vortex Interactions about a Generic Missile Airframe," Paper to be presented at AIAA SciTech 2022, 2022.

[5] Anderson, M., et al., "The Influence of the Numerical Scheme in Predictions of Vortex Interaction about a Generic Missile Airframe," Paper to be presented at AIAA SciTech 2022, 2022. 
[6] Shaw, S., et al., "The influence of modelling in predictions of vortex interactions about a generic missile airframe: RANS," Paper to be presented at AIAA SciTech 2022, 2022.

[7] Tormalm, M., et al., "The Influence of Scale Resolving Simulations in Predictions of Vortex Interaction about a Generic Missile Airframe," Paper to be presented at AIAA SciTech 2022, 2022.

[8] Loupy, G., "A Focused Study into the Prediction of Vortex Formation about Generic Missile and Combat Aircraft Airframes," Paper to be presented at AIAA SciTech 2022, 2022.

[9] Schnepf, C., and Tormalm, M., "Comparisons of predicted and measured aerodynamic characteristics of the DLR LK6E2 missile airframe," Paper to be presented at AIAA SciTech 2022, 2022.

[10] DeSpirito, J., et al., "Comparisons of Predicted and Measured Aerodynamic Characteristics of the DLR LK6E2 Missile Airframe (Scale Resolving)," Paper to be presented at AIAA SciTech 2022, 2022.

[11] Taylor, N., "AVT-316 Missile Facet: 316 Missile Facet: Lessons learned concerning the prediction of Vortex Flow Interactions about Generic Missile Configurations," Paper to be presented at AIAA SciTech 2022, 2022.

[12] Spalart, P., "Strategies for turbulence modelling and simulations," International Journal of Heat and Fluid Flow, Vol. 21, No. 2, 2000.

[13] Steijl, R., Barakos, G. N., and Badcock, K., "A framework for CFD analysis of helicopter rotors in hover and forward flight," International Journal for Numerical Methods in Fluids, Vol. 51, No. 8, 2006, pp. 819-847.

[14] Steijl, R., and Barakos, G. N., "Sliding mesh algorithm for CFD analysis of helicopter rotor-fuselage aerodynamics," International Journal for Numerical Methods in Fluids, Vol. 58, No. 5, 2008, pp. 527-549.

[15] Osher, S., and Chakravarthy, S., "Upwind schemes and boundary conditions with applications to Euler equations in general geometries," Journal of Computational Physics, Vol. 50, No. 3, 1983, pp. 447-481.

[16] van Leer, B., "Towards the ultimate conservative difference scheme. V. A second-order sequel to Godunov's Method," Journal of Computational Physics, Vol. 32, No. 1, 1979, pp. 101-136.

[17] van Albada, G. D., van Leer, B., and Roberts, W. W., “A Comparative Study of COmputational Methods in Cosmic Gas Dynamics," Astronomy and Astrophysics, Vol. 108, No. 1, 1982, pp. 76-84.

[18] Axelsson, O., Iterative Solution Methods, Cambridge University Press, 1994.

[19] Menter, F., “Two-Equation Eddy-Viscosity Turbulence Models for Engineering Applications," AIAA Journal, Vol. 32, No. 8, 1993, pp. 1598-1605.

[20] Hellsten, A., "New Advanced k-omega Turbulence model for High-Lift Aerodynamics," AIAA Journal, Vol. 43, No. 9, 2005 , pp. 1857-1869.

[21] Menter, F., Kuntz, M., and Bender, R., "A scale-adaptive simulation model for turbulent flow predictions," Proceedings of the 41th AIAA Aerospace Sciences Meeting and Exhibit, 2003.

[22] Menter, F., and Egorov, Y., "The Scale-Adaptive Simulation Method for Unsteady Turbulent Flow Predictions. Part 1:Theory and Model Ddescription," Flow, Turbulence and Combustion, Vol. 85, No. 1, 2010, pp. 113-138.

[23] “NASA Langley Research Center Turbulence Modeling Resource,”, ????

[24] Roache, P. J., "Perspective: A Method for Uniform Reporting of Grid Refinement Studies," Journal of Fluids Engineering, Vol. 116, No. 3, 1994, pp. 405-413.

[25] Roache, P. J., “Verification of Codes and Calculations,” AIAA Journal, Vol. 36, No. 5, 1998, pp. 696-702.

[26] “Vortex Interaction Effects Relevant to Military Air Vehicle Performance," https://www.sto.nato.int/Lists/test1/ activitydetails . aspx?ID=16476s 2021.

[27] “ANSYS ICEM CFD HEXA,”https://www . ansys.com/products/fluids 2021. 